\title{
edmetic
}

Revista de Educación Mediática y TIC

\section{Matemáticas y TIC, juntas pero no revueltas}

El desarrollo de los Reales Decretos que regulan la puesta en marcha de la Educación Primaria y Secundaria en España, plantean dos competencias básicas bien diferenciadas, de un lado encontramos la matemática y de otro la digital.

En niveles superiores de la enseñanza, ambas se convierten en el sustento y desarrollo de conocimientos más complejos, a veces de difícil comprensión por parte de los estudiantes, que harán que aspectos de su vida cotidiana cobren sentido.

Por separado las dos tienen su propia identidad. Numerosos son los trabajos e investigaciones realizados por los prácticos y teóricos de la educación matemática, que alaban las bondades de dicha materia y la necesidad de hacer ver su lado más positivo, lejos de complejidades y dificultades terminológicas.

Desde hace relativamente poco tiempo, este concepto se ha ido identificando con "alfabetización matemática". Este es entendido por algunos autores como Rico $(2004,2)$ como "la capacidad individual para identificar y entender el papel que las matemáticas tienen en el mundo, hacer juicios bien fundados y usar e implicarse con las matemáticas en aquellos momentos en que se presenten necesidades en la vida de cada individuo como ciudadano constructivo, comprometido y reflexivo". Dicha comprensión aboga, desde mi punto de vista, por la unificación con otros medios, materias y recursos que reviertan en una mayor comprensión terminológica de conceptos, principios, 
leyes, etc.

Sentadas las bases de una buena enseñanza matemática en niveles primarios de la formación, la educación superior podrá afianzar dicha competencia.

Por otra parte, encontramos el interés que desde hace años los gobiernos en general tienen por una alfabetización digital de la ciudadanía, por ello si se aúnan ambas estaremos logrando compensar deficiencias en ambos campos y, además, abriendo nuevas vías de investigación.

Si como binomio de trabajo diario de aula tomamos Matemáticas-TIC, podremos encontrar numerosas aportaciones que desarrolladas desde y en la red ayudaran tanto a los profesionales de la educación como a los estudiantes y progenitores. Un ejemplo de ello lo encontramos en http://www.disfrutalasmatematicas.com/, http://sauce.pntic.mec.es/jdiego/ o http://www.matesymas.es/ donde alumnado y profesorado podrá de manera amena y divertida "practicar" las mates. A nivel internacional podemos encontrar el blog http://matematicasecundariaceicelaya.blogspot.com.es/, de reciente creación, pero no por ello menos valioso creado desde México, http://arlitaquirozrodas.blogspot.com.es/ en Perú.

Para una neófita del mundo de los números en particular y del universo matemático en general, en este número de EDMETIC podré/podremos encontrar una nueva perspectiva de dos contenidos y competencias básicas, que me/nos ayudaran por una parte a comprender y por otra a perder el miedo a un contenido que, como ya hemos mencionado anteriormente, a veces se me antoja y se me ha antojado abrupto.

Las experiencias que hasta aquí traen los autores tanto nacionales como internacionales ponen de relieve la gran variedad de aportaciones que Matemáticas-TIC y TIC-Matemáticas tienen hoy.

Agradecer a los firmantes de este segundo volumen en nuestro primer año de vida.

Verónica Marín-Díaz

Editora de EDMETIC, Revista de Educación Mediática y TIC 
vmarin@uco.es

\section{Referencias Bibliográficas}

Rico, L. (2004). Reflexiones sobre la formación inicial del profesor de matemáticas en secundaria. Profesorado, Revista de Curriculum y Formación de profesorado, 8 (1), Recuperado de: http://www.ugr.es/ recfpro/rev81 ART2.pdf. 